\title{
ON THE PARSING OF LI-REGUIAR GRAMMARS
}

\author{
Anton Nijholt \\ Department of Mathematics \\ Free University, Amsterdam, The Netherlands*
}

\section{INPRODUCTION}

Culik II and Cohen [1] introduced the class of LR-regular grammars, an extension of the $\mathrm{LR}(\mathrm{k})$ grammars. In [2] and [3] the same idea is applied to the class of $L I(k)$ grammars and the LL-regular grammaxs were introduced. The idea is that the parsing is done with a two-scan parsing algorithm. The first scan of a sentence w to be parsed, called the pre-scan, is done by a Moore machine (reading from right to left) and yields a string of symbols which is the input for a deterministic pushdown transducer (dpdt). In the case of an IR-regular grammar $G$ the result of the pre-scan is a sentence of an $L R(0)$ grammar $G^{\prime}$ which can be constructed from $G$, and the parsing can be done with regard to this LR(O) grammar. In the case of an IIregular gramnar it is possible to construct a strict deterministic grammar [8] and after the pre-scan has been performed the parsing can be done with regard to this grammar. However a more efficient method can be given since it can be shown that the parsing can be done with a 1-predictive parsing algorithm or even with a simple II(1) parsing method (see section 3 and [2]).

The classes of LR-regular and LImregular grammars have some similar properties as the classes of $L R(k)$ and $L I(k)$ grammars. Moreover, sometimes the proofs of these properties need only slight adaptions. In this paper the proofs are omitted. In [2] proofs, and some properties and examples not given here, can be found. In the remainder of this section we give some notations and definitions. In section 2 we list some properties and the main part of this paper is in section 3 where we consider the parsing of Li-regular grammars.

A (reduced) context-free Eramar $(\mathrm{cfg})$ is denoted by $G=(\mathrm{N}, \mathrm{T}, \mathrm{P}, \mathrm{S}), \mathrm{V}=\mathrm{N} \cup \mathrm{T}$; we will denote elements of $N$ by $A, B, C, \ldots$; elements of $T$ by $a, b, c, \ldots$; elements of $T^{*}$ by $\ldots w, x, y, z ;$ elements of $V^{*}$ by $\alpha, \beta, \gamma, \delta, \ldots ; \varepsilon$ denotes the empty string. A regular partition of $\mathrm{T}^{*}$ is a partition of $\mathrm{T}^{*}$ of finite index and such that each block is a regular set. The states of a Moore machine (with input alphabet I') define a regular partition of $\mathrm{T}^{*}[4]$.

\footnotetext{
* The research reported in this paper has been carried out at the Department of
} Applied Mathematics of the Twente University of Tekhnology, Enschede. 
The following definition can be found in [6] and in a generalized form in [5].

\section{DEFINITION 1. (Left-cover)}

Let $G$ and $G^{\prime}$ be cfgs, $G=(N, T, P, S), G^{\prime}=\left(N^{\prime}, T, P^{\prime}, S^{\prime}\right)$ and $L(G)=L\left(G^{\prime}\right)$. $G^{\prime}$ leftcovers $G$ if there is a homomorphism h from $P^{\prime}$ to $P^{*}$ (extended to $P^{\prime *}$ ) such that

(1) if $S^{\prime} \stackrel{\frac{\rho^{\prime}}{L}}{\longrightarrow} w$, then $S \frac{h\left(\rho^{\prime}\right)}{\ell} w$, and

(2) for all $\rho$ such that $S \frac{\rho}{l} w$, there exists $\rho^{\prime}$ such that $S^{\prime} \stackrel{\rho^{\prime}}{l} w$ and $h\left(p^{\prime}\right)=p$.

In this definition $\rho$ and $\rho^{\prime}$ denote the concatenations of the productions used in the left-most derivations.

\section{II-REGULAR GRAMMARS, AN IXTENSION OF LI( $\mathrm{k}$ ) GRAMMARS}

\section{DEFINITION 2. (IL-regular grammar)}

Let $G=(N, T, P, S)$ be a efg, $\pi$ a regular partition of $T$. G is said to be an $I I(\pi)$ gremmar if, for any two left-most derivations of the forms

$$
\begin{aligned}
& \text { (i) } \mathrm{S} \stackrel{*}{=} \mathrm{wA \alpha} \rightleftharpoons \mathrm{l}=\mathrm{w} \alpha \stackrel{\star}{\Longrightarrow} \mathrm{wx} \text {, } \\
& \text { (ii) } S \stackrel{\star}{l} \text { wÁ } \Longrightarrow \text { wô } \stackrel{\star}{\Longrightarrow} \text { wy, }
\end{aligned}
$$

where $\mathrm{x} \equiv \mathrm{y}(\bmod \pi)$, then we may conclude $\gamma=\delta$. A efg $G$ is said to be LL-regular or LLR if there exists such a partition $\pi$ of $\mathbb{T}^{*}$.

The class of grammars introduced in [3] is in fact a subclass of our class of ILR grammars. We prefer to call those grammars strong IIR grammars to obtain a framework analogous to the $L L(x)$ and strong $L(k)$ grammars. If we replace in definition 2 each occurrence of $w$ and $\alpha$ in ( $i$ ) by $w_{1}$ and $\alpha_{1}$ and in (ii) by $w_{2}$ and $\alpha_{2}$ respectively, then we obtain the definition of a strong LI( $\pi)$ gramar. It will be clear that every strong LLR gramar is LLR and easily can be verified that every $L I(k)$ grammar is LLR.

Example 1. Cfg $G$ with only productions $S \rightarrow a A a a|b A b a a| b A b a b$ and $A \rightarrow b A \mid b$ is neither LI nor strong LIR, However $G$ is LIR. A regular partition for $G$ is given in section 3.

\section{THEOREM 1.}

a. Every LLR grammar is unambiguous

b. No LIR grammar is left-recursive

c. It is decidable whether a $\mathrm{cg}$ is $L L(\pi)$ for a given regular partition $\pi$.

Since every left-recursive grammar can be covered by a non-left-recursive grammar [7] in some cases it may be useful to see if elimination of left recursion yielas an $\mathrm{LU}(\pi)$ gramar for some regular partition $\pi$. Theorem $1 \mathrm{c}$. can be proved in a way such that it amounts to the construetion of the parsing algorithm. This algorithm will be 
discussed in the following section.

The following two theorems have proofs which differ only in details of proofs for $\mathrm{LW}(\mathrm{k})$ and $\mathrm{LR}(\mathrm{k})$ gramars as given in [6].

THEOREM ?.

Every $L I(\pi)$ gramar, where $\pi$ is a left congruence, is an $L R(\pi)$ gramar.

Since a left congruence can always be found by refining of the partition we may say that every ILR grammar is also an LRR grammar. This inclusion is proper.

Example 2. Cfg G with only production $S \rightarrow C c, C \rightarrow C b \mid b$ is $L R(O)$ and hence LRR, but $G$ is not LLR.

THEOREM 3.

Every LLR gramar $G$, such that $\varepsilon \notin I(G)$, has an equivalent LLR grammar $G^{\prime}$ in Greibach normal form (GNF'). Moreover G' left-covers $G$.

Like the equivalent theorem for $\mathrm{LL}(\mathrm{k})$ grammars this theorem is useful in showing that a language may be non-deterministic, The LLR languages are properly contained in the LRR languages. For example, the language $I=\left\{c^{n} d^{n}, c^{n+1} d^{n} \mid n>1, I \geq 1\right\}$ is a deterministic language, and therefore LRR, but it has no LLR grammar in GNF.

\section{PARSING OF LL-REGUALR GRAMMARS}

An LI-regular grammar can be parsed, after a regular pre-scan from right to left has been performed, by using a strict deterministic parsing method [2]. This section however is devoted to a generalization of the LL(k)-parsing method. This generalization is such that any $L L(\pi)$ grammar can be parsed, apter a regular pre-scan from right to left has been performed, with a 1-predictive parsing algorithm.

First we need the following definition, in which $\pi$ is a regular partition of $\mathbb{T}^{*}$, $\pi=\left\{B_{0}, B_{1}, \ldots, B_{n}\right\}$ and $\alpha \in V^{*}$.

DEFINITION 3.

BLOCK $(\alpha)=\left\{B_{k} \in \pi \mid L(\alpha) \cap B_{k} \neq \emptyset\right\}$. If $B_{i}, B_{j} \leqslant \pi$, then $B_{i} \square B_{j}=\left\{B_{k} \in \pi \mid B_{k} \cap\left(B_{i} \cdot B_{j}\right) \neq \emptyset\right\}$, where $B_{i} \cdot B_{j}$ denotes the usual concatenation of sets of strings.

Let $I_{1}, L_{2} \leq \pi$, then $L_{1} \square I_{2}=\left\{B_{k} \in \pi \mid B_{k} \in B_{i} \square B_{j}, B_{i} \in I_{1}\right.$ and $\left.B_{j} \in I_{2}\right\}$.

Notice that $L(\alpha)$ is a context-free language (cfl), $B_{k}$ is a regular set and therefore $L(\alpha) \cap B_{k}$ is a cfl. Hence $i t$ is decidable whether $L(\alpha) \cap B_{k}$ is non-empty [4]. This definition, together with lemma 1 we will give below, enables us to introduce the generelized parsing method.

IEMMA 1.

a. $\operatorname{BLOCK}(\alpha \beta)=\operatorname{BLOCK}(\alpha) \square \operatorname{BLOCK}(\beta)$.

b. Let $G=(\mathbb{N}, T, P, S)$ be a efg and suppose $A \rightarrow \beta$ and $A \rightarrow \gamma$ are in $P, \beta \neq \gamma$. G is not 
$L L(\pi)$ iff there is a derivation $S \stackrel{*}{l} \rightarrow w A \alpha$ and

$(\operatorname{BLOCK}(\beta) \square \operatorname{BLOCK}(\alpha)) \cap(\operatorname{BLOCK}(\gamma) \square \operatorname{BLOCK}(\alpha)) \neq \varnothing$.

Analogous to the theory of $L I(k)$ parsing we define functions $T_{A, I}$ on partition $\pi$ (these functions are called the LL( $\pi$-tables), where $A$ is a nonterminal and $L$ is a set of blocks. These functions satisfy the following conditions.

(1) $T_{A, I}\left(B_{K}\right)=$ exror, if there is no production $A \rightarrow \alpha$ in $P$ such that $B L O C K(\alpha) \square I$ contains $B_{k}$.

(2) $\mathrm{T}_{\mathrm{A}, \mathrm{I}}\left(\mathrm{B}_{\mathrm{K}}\right)=\left(A \rightarrow \alpha,\left[\mathrm{L}_{1}, \mathrm{I}_{2}, \ldots, \mathrm{L}_{\mathrm{m}}\right]\right)$, if $\mathrm{A} \rightarrow \alpha$ is the unique production in $\mathrm{P}$ such that $B L O C K(\alpha) \square I$ contains $B_{k}$. If $\alpha=x_{0} C_{1} x_{1} C_{2} \ldots C_{m} x_{m}, m \geq 0, C_{i} \in \mathbb{N}$ and $x_{i} \in T^{*}$, then $L_{i}=\operatorname{BLOCK}\left(x_{i} C_{i+1} \cdots C_{m} x_{m}\right) \square L,(0 \leq i \leq m)$.

(3) $T_{A, L}\left(B_{K}\right)=$ undefined if there are two or more productions $A \rightarrow \alpha_{1}$ and $A \rightarrow \alpha_{2}$, $\alpha_{1} \neq \alpha_{2}$ such that $\left(\operatorname{BLOCK}\left(\alpha_{1}\right) \square L\right) \cap\left(\operatorname{BLOCK}\left(\alpha_{2}\right) \square L\right)$ contains $B_{k}$.

Now it will be clear that if efg $G$ is $\operatorname{LL}(\pi)$ and there is a derivation $S \stackrel{\star}{\stackrel{l}{l}} W A \alpha \stackrel{\star}{\Longrightarrow} w x$, then $T_{A, L}\left(B_{k}\right)$, where $x \in B_{K}$ and $L=B L O C K(\alpha)$, wiIl uniquely determine which production is to be used to expand $A$.

Starting with $L I(\pi)$-table $T_{0}=T_{S},\left\{B_{0}\right\}$, where $B_{0}=\{\varepsilon\}$, it is possible to determine the set $T(G)$ of all relevant $L L(\pi)$-tables of $G$. In the example at the end of this section $T(G)$ is given for the efg of example 1 .

With the LL( $\pi$ )-tables as input the following algorithm constructs a 1-predictive parsing table.

In this algorithm we use the partition $\pi_{0}=\left\{a T^{*} \mid a \in T\right\} \cup\{\varepsilon\}$ and we require that partition $\pi$ for which the parsing table is constructed is a refinement of $\pi_{0}$. We let $\pi=\left\{B_{0}, B_{1}, \ldots, B_{n}\right\}$, where $B_{0}=\{\varepsilon\}$. It is always possible to obtain such a partition $\pi$ if $\mathrm{G}$ is LL.L. The condition $\pi \subseteq \pi_{0}$ is introduced to prevent the parsing algorithm (see algoritm 2) from giving left parses for sentences which do not belong to $L(G)$. To each block in $\pi$ we assign a unique number $(0,1,2, \ldots, n)$, and we let $\pi$ also denote the set of these numbers. These numbers will be the output alphabet of the Moore machine in the parsing algorithm.

To each production in $P$ we also assign a unique number and we let $P$ also denote the set of these numbers.

ALGORITHM 1. (construction of a i-predictive parsing table) Input: $L L(\pi)$ gramar $G=(\mathbb{N}, T, P, S), \pi \subseteq \pi_{0}$ and the set $T(G)$. Output: a parsing table $Q$ for $G$,

Q: $(T(G) \cup T \cup\{\$\}) \times \pi \rightarrow\left((T(G) \cup T)^{*} \times P\right) \cup\{$ pop, accept, error $\}$

Method:

(1) if $A \rightarrow x_{0} C_{1} x_{1} C_{2} x_{2} \ldots C_{m} x_{m}$ is the $i$-th production in $P$ and $T_{A, I}$ is in $T(G)$, then for every $B_{j}$ such that $T_{A, L}\left(B_{j}\right)=\left(A \rightarrow x_{0} C_{1} x_{1} C_{2} x_{2} \ldots C_{m} x_{m},\left[L_{1}, L_{2}, \ldots, L_{m}\right]\right)$ we have $Q\left(T_{A, I}, j\right)=\left(x_{0} T_{C_{1}}, I_{1} x_{1} T_{C_{2}}, I_{2} x_{2} \cdots T_{C_{m}}, I_{m} x_{m}, \dot{i}\right)$.

(2) $Q(a, j)=p o p$, if $w \in B_{j}$ implies that the first symbol of $w$ is $a$. 
(3) $Q(\$, 0)=$ accept

(4) otherwise $Q(X, j)=$ error, for $X$ in $T(G) \cup T \cup\{\$\}$ and block $B_{j}$.

Now we are prepared to give the parsing algorithm. We let $w^{R}$ denote the string $w$ in a reversed order, $B_{j}^{R}=\left\{w^{R} \mid w \in B_{j}\right\}$ and $\pi^{R}=\left\{B_{j}^{R} \mid B_{j} \in \pi\right\}$. For convenience we assume that $G$ is $\operatorname{LI}(\pi)$, where $\pi$ is a left congruence. We assume the reader is familiar with the construction of a Moore machine $M_{\pi}$ which defines by its states the right congruence $\pi^{R}$. $M_{\pi}$ will perform the pre-scan from right to left.

ALGORITHM 2. (1-predictive parsing algorithm)

Input: $L I(\pi)$ gramar $G=(N, T, P, S)$, parsing table $Q$ and Moore machine $M_{\pi}$. The string $w=a_{0} a_{1} \ldots a_{i} a_{i+1} \ldots a_{m} \in T^{*}$ has to be parsed.

Output: The left parse for $w$ if $w \in L(G)$, otherwise 'error'.

Method:

(1) Apply $M_{\pi}$ to $w^{R}$ such that if $a_{i} a_{i+1} \cdots a_{m}$ is in block $B_{j}$, then the to $B_{j}^{R}$ corresponding state of $M_{\pi}$ gives output $j$. The result is a string $w_{\pi}=j_{0} j_{1} \cdots j_{m} \in \pi^{*}$.

(2) A configuration is a triple $(x, X \alpha, \psi)$, where

i. $x$ represents the unused portion of the original input string $w_{\pi}$.

ii. Xa represents the string on the pushdow list (with $X$ on top), $X \alpha \in(T(G) \cup T)^{*} \phi$.

iii. $\psi$ is the string on the output tape.

The initial configuration is $\left(w_{\pi}, T_{0} \$, \varepsilon\right)$, where $T_{0}=T_{S},\left\{B_{0}\right\}$, the accept configuration is $(\varepsilon, \$, \rho)$ where $\rho$ is the left parse of with respect to $G$.

(3) A move $r$ is defined on the configurations as follows:

i. $\left(j x, T_{k} \alpha, \psi\right) \vdash(j x, B \alpha, \psi i), T_{k} \in T(G)$ and $Q\left(T_{k}, j\right)=(\beta, i)$.

ii. $(j x, a \alpha, \psi) \vdash(x, \alpha, \psi), a \in T$ and $Q(a, j)=$ pop.

If none of these moves can be done, hence $Q(X, j)=$ error, then the parsing ceases.

Example 3. Cifg $G$ with only productions $1.5 \rightarrow$ aAaa, $2.5 \rightarrow$ bAbaa, $3 . \mathrm{S} \rightarrow$ bAbab, 4. $A \rightarrow b A$ and $5 . A \rightarrow b$. The table below gives a regular partition for $G$ which satisfies the conditions of the two algorithms.

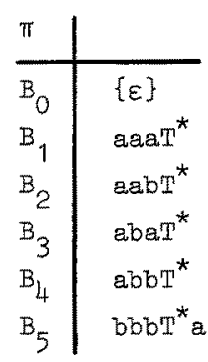

\begin{tabular}{l|l} 
& \\
\hline$B_{12}$ & $\mathrm{babT}^{*} \mathrm{~b}$ \\
$\mathrm{~B}_{13}$ & $\{\mathrm{~b}\}$ \\
$B_{14}$ & $\{\mathrm{bb}\}$ \\
$B_{15}$ & $\{\mathrm{bbb}\}$ \\
$B_{16}$ & $\{$ bba $\}$ \\
$B_{17}$ & $\{$ baa $\}$
\end{tabular}


In the II( $\pi)$-tables we only display the non-error entries.

\begin{tabular}{l|l}
$\mathrm{T}_{0}$ & $={ }_{\mathrm{S}},\left\{\mathrm{B}_{0}\right\}$ \\
\hline $\mathrm{B}_{3}$ & $\mathrm{~S} \rightarrow$ aAaa, $\left[\left\{\mathrm{B}_{21}\right\}\right]$ \\
$\mathrm{B}_{4}$ & $\mathrm{~s} \rightarrow \mathrm{aAa},\left[\left\{\mathrm{B}_{21}\right\}\right]$ \\
$\mathrm{B}_{5}$ & $\mathrm{~S} \rightarrow$ bAbaa, $\left[\left\{\mathrm{B}_{17}\right\}\right]$ \\
$\mathrm{B}_{6}$ & $\mathrm{~S} \rightarrow$ bAbab, $\left[\left\{\mathrm{B}_{18}\right\}\right]$
\end{tabular}

\begin{tabular}{l|l}
$T_{1}$ & $=T_{A,\left\{B_{21}\right\}}$ \\
\hline$B_{5}$ & $A \rightarrow b A,\left[\left\{B_{21}\right\}\right]$ \\
$B_{7}$ & $A \rightarrow b A,\left[\left\{B_{21}\right\}\right]$ \\
$B_{17}$ & $A \rightarrow b,[\varnothing]$
\end{tabular}

\begin{tabular}{l|l}
$T_{2}$ & $=T_{A,\left\{B_{17}\right\}}$ \\
\hline$B_{5}$ & $A \rightarrow b A,\left[\left\{B_{17}\right\}\right]$ \\
$B_{7}$ & $A \rightarrow b,[\phi]$
\end{tabular}

\begin{tabular}{l|l}
$T_{3}$ & $=T_{A,\left\{B_{18}\right\}}$ \\
\hline$B_{6}$ & $A \rightarrow b A,\left[\left\{B_{18}\right\}\right]$ \\
$B_{8}$ & $A \rightarrow b,[\varnothing]$
\end{tabular}

Parsing table Q. (only the entries of $\mathrm{T}_{0}, \mathrm{~T}_{1}, \mathrm{~T}_{2}$ and $\mathrm{T}_{3}$ are given)

\begin{tabular}{l|c|c|c|c|c|c|c|}
$\mathrm{a}$ & 3 & 4 & 5 & 6 & 7 & 8 & 17 \\
\hline $\mathrm{T}_{0}$ & $\mathrm{a} \mathrm{T}_{1} \mathrm{aa}, 1$ & $\mathrm{aT} \mathrm{T}_{1} \mathrm{aa}, 1$ & $\mathrm{bT}_{2} \mathrm{aa}, 2$ & $\mathrm{bT}_{3} \mathrm{bab}, 3$ & - & - & - \\
$\mathrm{T}_{1}$ & - & - & $\mathrm{bT}_{1}, 4$ & - & $b \mathrm{~T}_{1}, 4$ & - & $\mathrm{b}, 5$ \\
$\mathrm{~T}_{2}$ & - & - & $\mathrm{bT}_{2}, 4$ & - & $\mathrm{b}, 5$ & - & - \\
$\mathrm{T}_{3}$ & - & - & - & $b \mathrm{~T}_{3}, 4$ & - & $\mathrm{b}, 5$ & -
\end{tabular}

Let us apply algorithm 2 on $w=$ abbaa.

(1) applying $M_{\pi}$ yields $4.7 \cdot 17 \cdot 21.20$

(2) $\left(4.7 .17 .21 .20, \mathrm{~T}_{0} \phi, \varepsilon\right) \vdash\left(4.7 .17 .21 .20, \mathrm{aT}_{1} \mathrm{aa} \$, 1\right) \vdash\left(7.17 .21 .20, \mathrm{~T}_{1} \mathrm{aa} \phi, 1\right)$

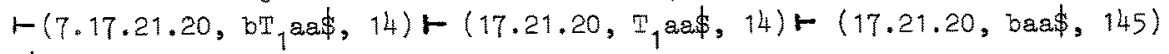
$\vDash(\varepsilon, \$, 145)$, and hence 145 is the left parse for abba.

Note. It is possible to show that if $C$ is in GNF then we can construct from parsing table $Q$ a simple $L L(1)$ grammar $G_{\pi}$ with properties:

(i) $\left[\left[M_{\pi}\left(w^{R}\right)\right]^{R} \mid w \in L(G)\right\} \subseteq L^{\prime}\left(G_{\pi}\right)$,

(ii) if $w \notin L(G)$ then $\left[M_{\pi}\left(w^{R}\right)\right]^{R} \notin L\left(G_{\pi}\right)$, and

(iii) there exist homomorphisms $h$ and $g$ such that if $\rho$ is a left parse for $w_{\pi} \in L\left(G_{\pi}\right)$ then $h(\rho)$ is a left parse for $w=g\left(w_{\pi}\right) \in L(G)$.

From these properties and from theorem 3 it follows that every LLR grammar can be parsed, after a regular pre-scan has been performed, with respect to a simple LL(1) grammar.

Acknowledgements :

I am grateful to prof. L. Verbeek who gave me the opportunity to do this work. 


\section{References.}

1. Culik II K, and Cohen R., IR-regular grammars - an extension of $L R(K)$ grammars, J. Comput. System Sei.7, (1973), No. 1, 66-96.

2. Nijholt A., Regular extensions of some classes of grammars, T.W. mem. No. 100, september 1975, Twente University of Technology.

3. Jarzabek S, and Krawczyk T., LI-regular gramars, Information Processing Letters, Vol. 4, No. 2, november 1975, 31-37.

4. Hopcroft J.E. and UlIman J.D., "Formal languages and their relation to automata", Add. Wesley, Reading, M.A., 1969.

5. Nijholt A., On the covering of parsable grammars, T.W. mem. No. 96 , september 1975, Twente University of Technology.

6. Aho A.V. and UlIman J.D., "The theory of parsing, translation and compiling", Vols. I and II, Prentice Hall, Englewood Cliffs, 1972 and 1973.

7. Nijholt A., On the covering of left-recursive gramars, T.W. mem. No. 127, april 1976, Twente University of Technology.

8. Harrison M.A. and Havel I.M., Strict deterministic grammars, J. Comput. System Sci. 7, (1973), No. 3, 237-277. 